\title{
Network Flow Models for Evacuation Planning
}

\author{
Urmila Pyakurel \\ Central Department of Mathematics, Tribhuvan University, Kathmandu, Nepal \\ Corresponding author: urmilapyakurel@gmail.com
}

Received: March 5, 2017 Revised: March 19, 2017 Accepted: March 22, 2017

\begin{abstract}
Because of increasing number of natural and human created disasters worldwide, a research on challenging issues of emergency management are being quite emerging for the betterment of today's complex real-life. The diversified operational research models and their solutions techniques together with their simulation schemes are yet not evident in realizing a generally acceptable solution of these challenging issues. We consider the planning problems in emergencies which is even unsolved yet in general. In this paper, we briefly give the models and algorithms on the existing solution approaches within the framework of evacuation planning network flow models, where the objectives are either to maximize the flow over time or minimize the time for transshipment of the estimated flow. The flow models and solution techniques with their insights presented here highlight the state of existing approaches and hint further direction of research in the field of evacuation optimization.
\end{abstract}

Keywords: Evacuation planning, network flow, contraflow

\section{Introduction}

The research in evacuation planning has been very much motivated due to the rapidly increased number of disasters, e.g. earthquakes, tsunamis, landslides, volcanic eruptions, hurricanes, typhoons, floods, terrorist attacks, chemical explosions, etc., world-wide. It is the process of shifting maximum number of evacuees from the disastrous areas to the safe destinations as quickly and efficiently as possible. Various mathematical models for flow maximization and time minimization have been studied in quite diversified research domains. The former one receives the maximum flow at the safe areas in given time, however, the latter one transships the given amount of evacuees in minimum time. Both objectives have been considered widely to solve different evacuation planning problems. However, an existence of integrated solution approach that could be accepted in wider range has been lacked. For details, we refer to $[1,3,9]$ and the references therein. 
The evacuation network is defined as a network that corresponds to a region (or a building) to be evacuated in which the intersections of streets (or rooms in a building) represent the nodes and the connections between these parts (i.e., streets in region, or doors between rooms) denote the edges. The initial locations of evacuees are the source nodes and the locations at safety regions are the sink nodes. The nodes and edges are bounded by capacities. Each arc has transit time or cost function. The group of evacuees that passes through the network over time is modeled as a flow. The plan is dependent upon the number of sources, sinks, parameters on the arcs and nodes, like constant, time-dependent or flow dependent capacities or transit times as well as additional constraints. The time may be discrete or continuous.

The maximum dynamic single-source single-sink flow model was introduced in [6] that maximizes a flow from a source to a sink in a given time horizon. This model can be used in evacuation planning by sending as many evacuees as possible from a source (dangerous node) to a sink (safe node) in a given time horizon. Author in [8] introduced the earliest arrival flow model. Their approach sends the maximum number of evacuees at every time point from the beginning. This model is very important for evacuation planning because it does not need the predetermined time horizon. If the supplies and demands are fixed on sources and sinks, respectively, then the earliest arrival flow model turns into the earliest arrival transshipment flow model [1].

The lex-maximum dynamic flow model was introduced in [10] that send the maximum amount of evacuees from sources to sinks in given priority. Priority orderings play quite important roles in emergency evacuation because of the level of disastrous situation and the relative weakness of the evacuees. Based on the problem of [6], the quickest flow model in which given amount of evacuees can be shifted in minimum time has been introduced in [2] and extended in [10]. The algorithms they presented are efficient. An optimal solution to the earliest arrival flow model follows the optimality of both the maximum flow and the quickest flow models. The natural transformation of [5] can transform all the discrete time models into continuous time models and vice versa.

Recently, the dynamic flow models are extended to the dynamic contraflow models in $[4,11,12,13,14,15,16,17,18]$. It is a widely accepted model. It minimizes the congestion. It increases the outbound roads capacities by reversing the direction of arcs or paths towards the sinks from the sources. Through the network with increased capacity, contraflow problem shifts the maximum number of evacuees to the sinks and decreases the evacuation time as well. It seeks to remove traffic jams and makes the traffic systematic and smooth. It is a very challenging issue of finding a network reconfiguration with ideal lane directions satisfying the given constraints to optimize the given objective. However, in this study, we only focus on network flow models and its solution procedures for evacuation planning.

The paper is organized as follows. We state the basic concepts and denotations for this paper in Section 2. Section 3 gives a short summery of existing static flow models for evacuation planning. Section 4 presents the dynamic flow models developed for evacuation planning. We 
summarize the solution techniques to solve the static and dynamic flow models in Section 5. This work is concluded with Section 6.

\section{Basic Concepts and Denotations}

The evacuation planning problem is modeled with an evacuation network that consists a directed graph $G=(V, A)$, where $V$ denotes a finite set of nodes and $A$ denotes a finite set of arcs, together with set of parameters on the nodes and/or arcs. The nodes are categorized into a set of source nodes $S \subseteq V$, a set of intermediate nodes $I \subseteq V$ and a set of sink nodes $D \subseteq V$. We assume that the number of nodes and arcs are $|V|=n$ and $|A|=m$, respectively. As we are considering the contraflow network, a two way network configuration is allowed. If a network has only one source and only one sink, we represent them as $s$ and $d$, respectively. In order to simplify the problem within a boundary of efficient model, we make some assumptions. Let $A_{v}^{\text {out }}=\{(v, w) \in A\}$ and $A_{v}^{\text {in }}=\{(w, v) \in A\}$ be the sets of outgoing arcs and incoming arcs, respectively, for the node $v \in V$. Not stated otherwise, we assume that there are no any incoming arcs to source node $s$ and outgoing arcs from sink node $d$, i.e., $A_{d}^{\text {out }}=A_{s}^{\text {in }}=\emptyset$.

The network consists of nonnegative functions of arc capacities $b_{A}: A \rightarrow \mathcal{R}^{+}$, node capacities $b_{V}: V \rightarrow \mathcal{R}^{+}$and arc costs $c_{A}: A \rightarrow Z^{+}$or arc transit times $\tau: A \rightarrow Z^{+}$depending upon the problem nature. The arc capacities $b_{A}(e), e \in A$ represent the maximum units of evacuees that may enter the initial node of $\operatorname{arc} e$ per time period. Let $x^{r}(e)$ be the rate of flow that is the amount of flow entering the arc $e$ per time unit. The flow rate is bounded by arc capacity of that arc, i.e., $x^{r}(e) \leq b_{A}(e)$. The group of evacuees is modeled as a flow which passes through the network over time.

The node capacities $b_{V}(v), v \in V$ bound the amount of evacuees allowed to hold at node $v$. The time needed to travel one unit of evacuees on the arc $e=(v, \mathrm{w})$ from node $v$ to node $w$ is the transit time $\tau(e)$. That means, if one unit of flow starts to move from node $v$ at time $t$ with flow rate one, it will reach the node $w$ at time $t+\tau(e)$ with the same flow rate. The cost needed for sending one unit of evacuees through the $\operatorname{arc} e$ is $c_{A}(e)$. Generally, supply and demand at nodes are unknown. If the integer supply/demand at each source and sink is known, it is denoted by the vector $\mu(v)$ for all $v \in S \cup D$.

The collection of date $\mathcal{N}=\left(V, A, b_{A}, b_{V}, \tau, S, D, T\right)$ with predetermined finite time $T$ is represented as a dynamic network. By discarding the time factor from dynamic network, a static network is obtained. Time $T$ may be discrete or continuous within which evacuation process should be completed. In discrete setting, it is considered as $t=0,1, \ldots, T$. However, in continuous time setting, $t$ can take any value in $[0, T]$. Let $\mathcal{T}$ be a domain of time, i.e., $\mathcal{T}=$ $\{0,1, \ldots, T\}$ in a discrete model and $\mathcal{T}=[0, T]$ in a continuous model. This paper considers both time settings. 
Some time, we construct a two terminal network from the multi-terminal network by adding a super-terminal node $(*)$ and introducing arcs $\left(*, s_{i}\right)$ to each $s_{i} \in S$ with infinite capacity and zero transit time, and $\operatorname{arcs}\left(d_{i}, *\right)$ to each $d_{i} \in D$ with infinite capacity and transit time $-(T+1)$ for given time period $T$. This network is called the extended network and is denoted by $\mathcal{N}^{*}$.

\section{Static Flow Models}

Let $\mathcal{N}=\left(V, A, b_{A}, c_{A}, s, d\right)$ be a two-terminal static network. Note that its arc capacity limits the total amount of flow on an arc. For example, the capacity limits the total number of vehicles on a street. Let a non-negative function $x_{\text {stat }}: A \rightarrow \mathcal{R}^{+}$represents the static flow. The maximum static $s-d$ flow maximizes the flow value $\operatorname{val}\left(x_{\text {stat }}\right)$ in Objective (1) satisfying the flow conservation and capacity Constraints (2) and (3), respectively, [6,7].

$\operatorname{val}\left(x_{\text {stat }}\right)=\sum_{e \in A_{d}^{\text {in }}} x_{\text {stat }}(e)=\sum_{e \in A_{s}^{\text {out }}} x_{\text {stat }}(e)$

$\sum_{e \in A_{v}^{\text {in }}} x_{\text {stat }}(e)-\sum_{e \in A_{v}^{\text {out }}} x_{\text {stat }}(e)=0, \forall v \in V \backslash\{s, d\}$

$0 \leq x_{\text {stat }}(e) \leq b_{A}(e), \forall e \in A$

A circulation for a static flow $x_{\text {stat }}$ with value val $\left(x_{\text {stat }}\right)$ is formed with flow conservation at each node of network $\mathcal{N}$. It is obtained by adding an extra $\operatorname{arc}(d, s)$ with the value val $\left(x_{\text {stat }}\right)$. Thus, we have a static flow with value zero as a zero circulation. If we have fixed flow value $\operatorname{val}\left(x_{\text {stat }}\right)$ for a network including $\operatorname{cost} c_{A}(e)$ on each arc $e \in A$, then the problem is the minimum cost flow that shifts the given flow from source to sink with minimum cost $\sum_{e \in A} c_{A}(e) x_{\text {stat }}(e)$. The minimum cost flow problem with zero circulation is a minimum cost circulation flow problem.

Example 1 Consider an evacuation network with a single source $s$, a single sink $d$ and intermediate nodes $x$ and $y$ as shown in Figure 1(a). As each arc has capacity and cost function, the network is static. During evacuation process, we do not allow the flow towards the source, along the cycles and outwards from the sink. Thus the arcs towards the source and outwards from the sink are empty.

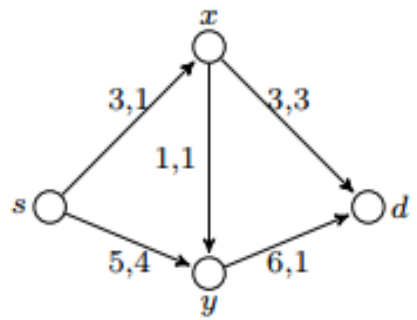

(a) (capacity, cost)

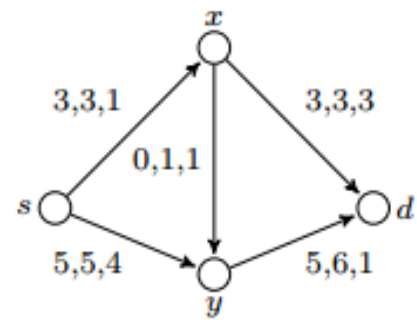

(b) (flow, capacity, cost)

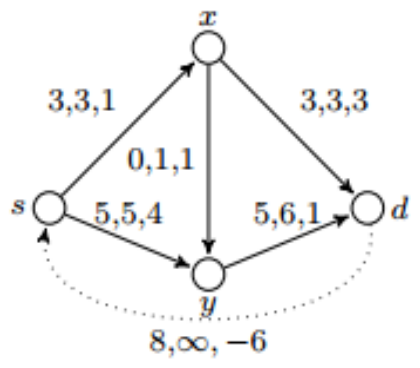

(c) (Circulation)

Fig. 1 (a) Evacuation network (b) Maximum flow (c) Minimum cost circulation flow 


\section{Dynamic Flow Models}

A two-terminal dynamic network $\mathcal{N}=\left(V, A, b_{A}, \tau, s, d, T\right)$ has capacity and transit times on each arc. In discrete model, the capacity limits the amount of flow that enters an arc at any point in time $\mathcal{T}=\{0,1, \ldots, T\}$. In continuous model, the capacity limits the rate of flow that enters an arc at any point in time $\mathcal{T}=[0, T]$. For example, the number of lanes on a street limits the number of vehicles that can enter it at the same time. If the cost function on each arc of Figure 1(a) is taken as transit time, then the network becomes a dynamic network. An arc between nodes $s$ and $x$ has capacity 3 and transit time 1 . If we assume that a time unit is 4 minutes, it takes 4 minutes for evacuees to travel from $s$ to $x$ and a maximum of 3 evacuees can simultaneously travel through the arc.

Let the non-negative functions $x_{\text {dyna }}: A \times \mathcal{T} \rightarrow \mathcal{R}^{+}$and $x^{r}: A \times \mathcal{T} \rightarrow \mathcal{R}^{+}$represent the dynamic flow in discrete model and the rate of flow on an $\operatorname{arc} e$ in continuous time model, respectively. Here $x^{r}(e, t)$ denotes the rate of flow that enters arc $e$ at continuous time $t$. We refer to $[6,7,2$, $9]$ for different studies on dynamic flow models.

\subsection{Maximum Dynamic Flow}

The maximum evacuation flow problem shifts the maximum possible number of evacuees from the disastrous areas to the safe destinations within a estimated time horizon $T$. The estimation of time horizon $T$ affects the problem directly. If $T$ is very long, the evacuation process may be slow and loss may be high. If $T$ is very short, all evacuees may not be shifted within the time. However, we consider $T$ as the total time of longest path of the network.

A maximum dynamic $s$ - $d$ flow $x_{d y n a}$ for given time $T$ maximizes the flow value $\operatorname{val}\left(x_{d y n a}, T\right)$ in Objective (4) satisfying the flow conservation and capacity Constraints (5-7). The inequality flow conservation constraint (6) allows to wait flow at intermediate nodes, however, the equality constraint (replace the inequality in (6) by equality) forces that flow entering an intermediate node must leave it again immediately.

$$
\begin{aligned}
& \operatorname{val}\left(x_{\text {dyna }}, T\right)=\sum_{\sigma=0}^{T} \sum_{e \in A_{s}^{\text {out }}} x_{\text {dyna }}(e, \sigma)=\sum_{\sigma=\tau(e)}^{T} \sum_{e \in A_{d}^{\text {in }}} x_{\text {dyna }}(e, \sigma-\tau(e)) \\
& \sum_{\sigma=\tau(e)}^{T} \sum_{e \in A_{v}^{i n}} x_{\text {dyna }}(e, \sigma-\tau(e))-\sum_{\sigma=0}^{T} \sum_{e \in A_{v}^{\text {out }}} x_{\text {dyna }}(e, \sigma)=0, \forall v \notin\{s, d\} \\
& \sum_{\sigma=\tau(e)}^{t} \sum_{e \in A_{v}^{i n}} x_{\text {dyna }}(e, \sigma-\tau(e))-\sum_{\sigma=0}^{t} \sum_{e \in A_{v}^{\text {out }}} x_{\text {dyna }}(e, \sigma) \geq 0, \forall v \notin\{s, d\}, t \in \mathcal{T} \\
& 0 \leq x_{\text {dyna }}(e, t) \leq b_{A}(e, t), \quad \forall e \in A, t \in \mathcal{T}
\end{aligned}
$$


Note that a maximum dynamic flow solution maximizes the flow in time $T$ and does not care the status at earlier time periods. This problem can be converted into minimum cost flow problem by using the transit time on each arc as the cost.

In continuous time, the maximum dynamic flow problem in given time horizon $T$ maximizes $\operatorname{val}\left(x^{r}, T\right)$ in Objective (8) satisfying the flow conservation and the flow rate capacity Constraint (9-11) which is similar to the discrete dynamic flow, with the sum over time replaced by an integral.

$$
\begin{aligned}
& \operatorname{val}\left(x^{r}, T\right)=\int_{0}^{T} \sum_{e \in A_{s}^{\text {out }}} x^{r}(e, t) d t=\int_{0}^{T} \sum_{e \in A_{d}^{\text {in }}} x^{r}(e, t-\tau(e)) d t \\
& \text { w.r.t. } \int_{0}^{t} \sum_{e \in A_{v}^{\text {in }}} x^{r}(e, t-\tau(e)) d t-\int_{0}^{t} \sum_{e \in A_{v}^{\text {out }}} x^{r}(e, t) d t \geq 0, \forall v \notin\{s, d\}, t \in \mathcal{T} \\
& \int_{0}^{T} \sum_{e \in A_{v}^{\text {in }}} x^{r}(e, t-\tau(e)) d t-\int_{0}^{T} \sum_{e \in A_{v}^{\text {out }}} x^{r}(e, t) d t=0, \quad \forall v \notin\{s, d\}, t \in \mathcal{T} \\
& 0 \leq x^{r}(e, t) \leq b_{A}(e, t), \quad \forall e \in A, t \in \mathcal{T}
\end{aligned}
$$

\subsection{Earliest Arrival Flow}

An earliest arrival flow (also known as universal maximum flow) problem on network $\mathcal{N}=$ $\left(V, A, b_{A}, \tau, S, D\right)$ demands a solution of maximal flow reaching the sinks for all points in time simultaneously. This problem captures the essence of the realistic evacuation planning, sending the maximum number of evacuees from the beginning of the evacuation process. It is a useful and reasonable property of network flow problems. The earliest arrival flow problem maximizes $\operatorname{val}\left(x_{\text {dyna }}, t\right)$ in (12) for all $t \in \mathcal{T}$ satisfying the constraints (5-7), [9].

$$
\operatorname{val}\left(x_{\text {dyna }}, t\right)=\sum_{\sigma=0}^{t} \sum_{e \in A_{s}^{\text {out }}} x_{\text {dyna }}(e, \sigma)=\sum_{\sigma=\tau(e)}^{t} \sum_{e \in A_{d}^{\text {in }}} x_{\text {dyna }}(e, \sigma-\tau(e))
$$

The earliest arrival flow satisfies the earliest arrival flow property, i.e., a cumulative amount of flows reaching the sinks in every considered time period and all preceding time periods of the considered one have to be maximal. Analogously, the flows leaving the sources have to be maximal.

As in discrete time setting, the earliest arrival flow problem in continuous time maximizes $\operatorname{val}\left(x^{r}, t\right)$ in Objective (13) satisfying the constraints (9) and (11) for all $t \in[0, T]$.

$$
\operatorname{val}\left(x^{r}, t\right)=\int_{0}^{t} \sum_{e \in A_{s}^{\text {out }}} x^{r}(e, t) d t=\int_{0}^{t} \sum_{e \in A_{d}^{i n}} x^{r}(e, t-\tau(e)) d t
$$




\subsection{Earliest Arrival Transshipment Flow}

Let $\mathcal{N}=\left(V, A, b_{A}, \tau, S, D, \mu(s), \vartheta(d)\right)$ be a multi-terminal network with a supply vector $\mu(s)$ for each $s \in S$ and a demand vector $\vartheta(d)$ for each $d \in D$. The multi-terminal earliest arrival flow problem sends total supply $\mu(S)=\sum_{s \in S} \mu(s)$ from the source set $S$ to the sink set $D$ with maximum value at each point of time $t>0$. If all supplies should be shifted within given time horizon $T$, then the problem turns into the transshipment problem. The earliest arrival transshipment problem maximizes $\operatorname{val}\left(x_{d y n a}, t\right)$ in Objective (14) satisfying the Constraints (5-7) for all $\in\{0,1, \ldots, T\}$.

$\operatorname{val}\left(x_{d y n a}, t\right)=\sum_{\sigma=0}^{t} \sum_{e \in A_{s}^{\text {out }}} x_{\text {dyna }}(e, \sigma)=\sum_{\sigma=\tau(e)}^{t} \sum_{e \in A_{d}^{\text {in }}} x_{\text {dyna }}(e, \sigma-\tau(e))=\mu(v), v=s, d$

Similarly, the earliest arrival transshipment problem in continuous time maximizes val $\left(x^{r}, t\right)$ in Objective (15) satisfying the Constraints (9) and (11) for all $t \in[0, T]$.

$\operatorname{val}\left(x^{r}, t\right)=\int_{0}^{t} \sum_{e \in A_{s}^{\text {out }}} x^{r}(e, t) d t=\int_{0}^{t} \sum_{e \in A_{d}^{\text {in }}} x^{r}(e, t-\tau(e)) d t=\mu(v), \quad v \in\{s, d\}$

\subsection{Quickest Flow}

If we have given flow value and we have to transship the flow value from sources to sinks in minimum time, then the problem will be quickest flow. It looks for a minimal time $\min T=$ $T\left(Q_{0}\right)$ such that flow value is at least $Q_{0}$ satisfying the Constraint (5), equality of the Constraint (6) and the capacity Constraint (7).

The quickest flow problem in continuous time transships the given $Q_{0}$ flow value in minimum time $\min T=T\left(Q_{0}\right)$ satisfying the equality of Constraint (9), Constraint (10), and the capacity Constraint (11).

\subsection{Lex-maximum Dynamic Flow}

For given time $T$ and an ordered set of multi-terminals with their priority, the maximum dynamic flow problem turns in to the lex-maximum dynamic flow problem. It finds a feasible flow that lexicographically maximizes the amount leaving each terminal in the given priority. Analogously, it maximizes the amount entering each terminal in the given priority.

If an ordered set of multi-terminals is given, then the maximum dynamic flow problem in given continuous time horizon $T$ is the lex-maximum dynamic flow problem. As in discrete time, it finds a feasible flow that lexicographically maximizes the amount leaving and entering each terminal in the given priority. 


\section{Solution Procedures of Network Flow Models}

\subsection{Residual Network}

Let $\mathcal{N}^{R}\left(x_{\text {stat }}\right)=(V, \vec{A} \cup \overleftarrow{A})$ with a static flow $x_{\text {stat }}$ be the residual network of $\mathcal{N}=(V, A$, $\left.b_{A}, \tau, s, d, T\right)$, where $\vec{A}=\left\{\vec{e}=e \mid x_{\text {stat }}(e)<b_{A}(e)\right\}$ be the set of forward arcs having capacity $b_{A}(e)-x_{\text {stat }}(e)$ and cost (transit time) $\tau(e)$ and $\overleftarrow{A}=\left\{\overleftarrow{e}=(\operatorname{head}(e)\right.$, tail $\left.(e)) \mid x_{\text {stat }}(e)>0\right\}$ be the set of backward arcs having capacity $x_{\text {stat }}(e)$ and a cost (transit time) $-\tau(e)$.

Example 2 If network has no residual flow, then obtained flow is a maximum. The maximum static flow through the network given in Figure 1 (a) of Example 1 is 8 with paths $s-x-d$ and $s-y-d$ by negating the cost function. If we include the cost, then the problem is minimum cost flow. To transship 8 units flow from $s$ to $d$ through paths $s-x-d$ and $s-y-d$, total 37 units cost requires that is the minimum cost. By adding an arc from sink $d$ to source $s$ with infinite capacity and $-(T+1)$ cost, where $T$ is the maximum length (cost) of path from source to sink, the problem turns into minimum cost circulation flow as shown in Figure 1(b). Here, the value of $T$ is 5 . When the capacity of the $\operatorname{arc}(d, s)$ is fixed as the maximum static flow value 8 , there exists a zero circulation.

For the static network with static flow value $\operatorname{val}\left(x_{\text {stat }}\right)=8$ through paths $s-x-d$ and $s-y-d$ of Figure 2(a), we construct its residual network as in Figure 2(b). There exists a path $s-x-y-d$ from source to sink through which we can increase flow value 1 in the residual network. This path is an augmenting path. Since, there exists no more augmenting paths in residual network, the obtained flow $\operatorname{val}\left(x_{\text {stat }}\right)+1=8+1=9$ is the maximum static flow through network in Figure 2(a). Notice that if the cost function on the arcs is reconsidered as transit time, then it will be the residual network of dynamic flow problem.

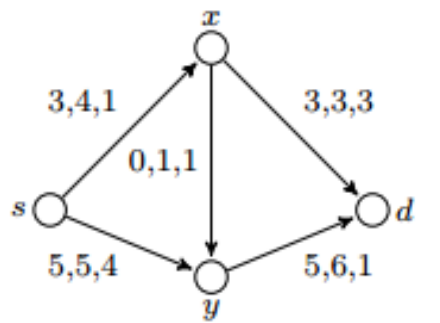

(a) (flow, capacity, cost)

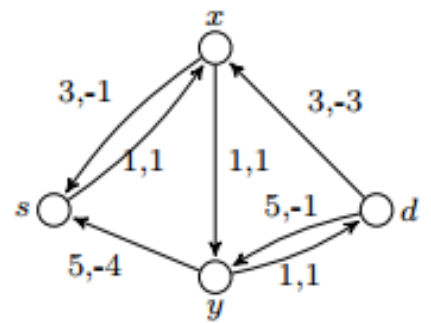

(b) (Residual capacity, cost)

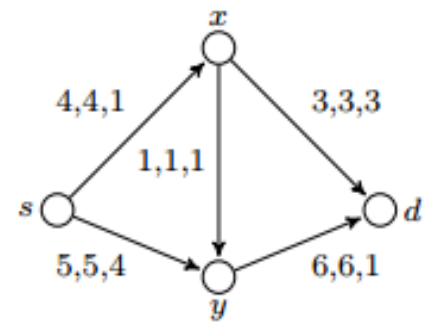

(a) (flow, capacity, cost)

Fig. 2 (a) Static flow network (b) Residual network, (c) Optimal flow network

\subsection{Time-expanded Network}

In order to solve the maximum dynamic flow problem, [6,7] introduced the time expanded network. It is defined as $\mathcal{N}(T)=\left(V_{T}, A_{M} \cup A_{H}\right)$, an expansion of the dynamic network, where each node $v$ of the static graph is copied $T$ times to obtain a node $v(t)$ for each $v \in V$ and each 
$t \in\{0,1, \ldots, T\}$. For each arc $e=(v, w) \in A$, the arc from $v(t)$ to $w(t+\tau(v, w))$ with capacity $b_{A}(v, w)$ is called movement arc and the arc from $v(t)$ to $v(t+1)$ with capacity $b_{V}(v)$ is called holdover arc which allow storage of flow at the nodes. The capacity of holdover arcs is infinite. If the holdover at nodes are not allowed, then holdover arcs will be omitted. Authors in $[6,7]$ proved a very important property as Theorem 1 which is the main root of the developed network flow models up to today.

Theorem 1 The maximum dynamic $s$ - $d$ flow in a given network $\mathcal{N}$ is equivalent to the maximal static $s_{0}-d_{T}$ flow in the time expanded network $\mathcal{N}(T)$.

If we compute the maximum dynamic flow of Figure 2(a) assuming cost as transit time and using time expanded network, same flow value can be computed as in Figure 3.

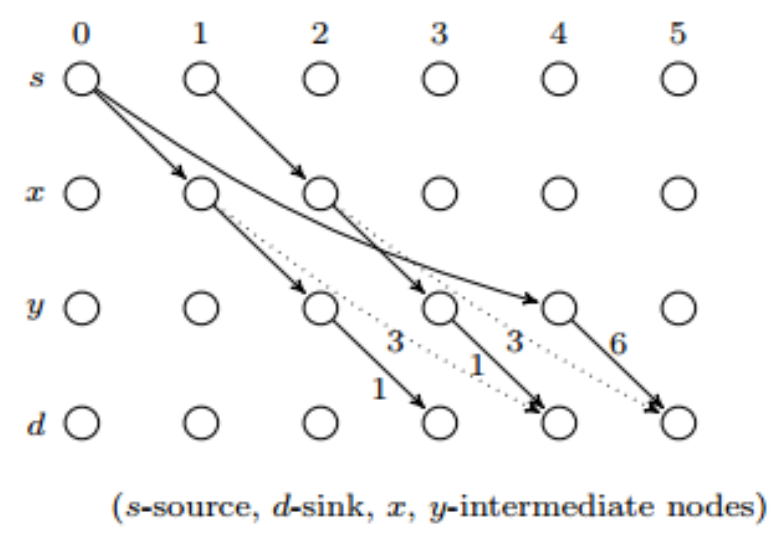

Fig. 3 Maximum static flow on time expanded network of Figure 2(a)

The network $\mathcal{N}(T)$ is static and depends directly upon the time $T$. Network $\mathcal{N}(T)$ is linear in $T$ having at most $O(n T)$ nodes and $O(n+m) T$ arcs. The concept of $\mathcal{N}(T)$ has been updated in most of the literatures, see for example, [9]. For large time horizon, the size of $\mathcal{N}(T)$ will be very large. This is a great disadvantage of the method though well-known classical static methods could be applied. All the algorithms based on $\mathcal{N}(T)$ are polynomial in $T$ and thus have pseudopolynomial running time.

\subsection{Flow Decomposition}

Let $P$ be a path, i.e., a sequence of nodes and arcs and let $\mathcal{P}=\left\{P_{1}, \ldots, P_{k}\right\}$ with $k \leq m$ be a finite set of paths from the source to the sink on a dynamic network $\mathcal{N}=\left(V, A, b_{A}, \tau, s, d, T\right)$. A path becomes a cycle if its end nodes are the same. Let $C$ be a cycle and $\mathcal{C}=\left\{C_{1}, \ldots, C_{k}\right\}$ with $k \leq m$ be a finite set of cycles. Let $x_{\text {stat }}\left(P_{k}\right)$ be the flow with value $\operatorname{val}\left(x_{\text {stat }}\left(P_{k}\right)\right)$ along path $P_{k} \in \mathcal{P}$ in the dynamic network. It holds $x_{\text {stat }}=\sum_{k=1}^{p} x_{\text {stat }}\left(P_{k}\right)$, where all paths in $\mathcal{P}$ start and end at the terminal nodes and use the arcs in the same direction as $x_{\text {stat }}$ does. This is called the 
standard chain decomposition [6,7]. If static flow $x_{\text {stat }}$ is decomposable into set of paths $\mathcal{P}$, the MSF problem can be formulated as

$$
\begin{aligned}
& \operatorname{maximize} \operatorname{val}\left(x_{\text {stat }}\right) \\
& \text { subject to } \sum_{P_{k} \in \mathcal{P}} \operatorname{val}\left(x_{\text {stat }}\left(P_{k}\right)\right)=\operatorname{val}\left(x_{\text {stat }}\right) \\
& \sum_{P_{k} \in \mathcal{P}: e \in P_{k}} \operatorname{val}\left(x_{\text {stat }}\left(P_{k}\right)\right) \leq b_{A}(e), \quad \forall e \in A
\end{aligned}
$$

A flow decomposition with zero flows on all cycles, known as a path decomposition, is also denoted by $\mathcal{P}$. One may assume that there is no flow along any cycle as the positive flow along all cycles could be canceled.

For example, in Figure 2, the maximum static flow value val $\left(x_{\text {stat }}\right)=9$ is decomposed into three paths $P_{1}=s-x-d$ with flow value $\operatorname{val}\left(x_{\text {stat }}\left(P_{1}\right)\right)=3, P_{2}=s-y-d$ with flow value $\operatorname{val}\left(x_{\text {stat }}\left(P_{2}\right)\right)=5$ and $P_{3}=s-x-y-d$ with flow value $\operatorname{val}\left(x_{\text {stat }}\left(P_{3}\right)\right)=1$ satisfying the capacity constraint, i.e., $\operatorname{val}\left(x_{\text {stat }}\left(P_{k}\right)\right) \leq b_{A}(e)$. Thus paths $P_{1}, P_{2}, P_{3} \in \mathcal{P}$ start and end at the same terminal nodes $s$ and $d$, and use arcs in same direction as $x_{\text {stat }}$ uses. Moreover, we have the final value calculated as $\operatorname{val}\left(x_{\text {stat }}\right)=\sum_{k=1}^{p} x_{\text {stat }}\left(P_{k}\right)=3+5+1=9$.

\subsection{Temporally Repeated Dynamic Flow}

A maximum dynamic flow can be obtained in temporally repeated flows, [7]. In process, the optimal static flow is decomposable into paths that are temporally repeated over given time horizon $T$, yielding the maximum dynamic flow. For a feasible static flow $x_{\text {stat }}$ and its path decomposition $\mathcal{P}$ with flow $x_{\text {stat }}$ along the path $P_{k} \in \mathcal{P}$, the associated temporally repeated flow sends $x_{\text {stat }}\left(P_{k}\right)$ flow units along the path $P_{k}$ for $\left(T-\tau\left(P_{k}\right)+1\right)$ times at periods $0,1, \ldots, T-$ $\tau\left(P_{k}\right)$, where $\tau\left(P_{k}\right)=\sum_{e \in P_{k}} \tau(e)$. The flow value of the temporally repeated flow is $\operatorname{val}\left(x_{\text {dyna }}, T\right)=\sum_{P_{k} \in \mathcal{P}}\left(T-\tau\left(P_{k}\right)+1\right) x_{\text {stat }}\left(P_{k}\right)$ from which we can derive the following important result.

$$
\begin{aligned}
\operatorname{val}\left(x_{\text {dyna }}, T\right) & =\sum_{P_{k} \in \mathcal{P}}\left(T-\tau\left(P_{k}\right)+1\right) x_{\text {stat }}\left(P_{k}\right) \\
& =(T+1) \operatorname{val}\left(x_{\text {stat }}\right)-\sum_{P_{k} \in \mathcal{P}} \sum_{e \in P_{k}} \tau(e) x_{\text {stat }}\left(P_{k}\right) \\
& =(T+1) \operatorname{val}\left(x_{\text {stat }}\right)-\sum_{e \in A} \tau(e) \sum_{P_{k}: e \in P_{k}} x_{\text {stat }}\left(P_{k}\right) \\
& =(T+1) \operatorname{val}\left(x_{\text {stat }}\right)-\sum_{e \in A} \tau(e) \cdot x_{\text {stat }}(e)
\end{aligned}
$$


Recall that, the maximum dynamic flow problem is equivalent to solving a minimum cost static flow problem with the arc costs as the arc transit time in both discrete and continuous time periods. Thus, the maximum temporally repeated flow can be obtained by finding a minimum cost circulation in the static network with an additional edge $(d, s)$ with infinite capacity and $-(T+1)$ cost, see also $[12,18]$.

For example, if we consider Figure 2(a) as a dynamic network where each arc has flow value, capacity and transit time, then the dynamic flow can be computed with temporally repeated paths in time $T=5$ as follows. First path $P_{1}=s-x-y-d$ reaches to sink at time 3 with flow value $x_{\text {stat }}\left(P_{1}\right)=1$ and it repeats at times 4 and 5 with same amount of flow. Second path $P_{2}=s-x-d$ reaches to sink at time 4 with flow value $x_{\text {stat }}\left(P_{2}\right)=3$ and it repeats again and reaches to sink at time 5 with same flow value. Similarly, third path $P_{3}=s-y-d$ reaches to sink at time 5 with flow units $x_{\text {stat }}\left(P_{3}\right)=5$. Thus in time 5 , total 14 units flow can be reached to the sink which is a maximum dynamic flow.

\section{Concluding Remarks}

The evacuation planning problems are the most important issues for saving the people and property, and supporting relief distribution within the emergency management strategy. These large scale real-life problems are computationally difficult to solve even approximately. Although few limited cases are solvable in polynomial time, their implementations do not reflect the desired general solutions. Most of the existing literature that deal with the heuristic and simulation approaches as exact solutions with real time settings are quite far from the reality of implementations. Researchers in diversified fields of science and technology, engineering, management and social sciences have contributed a bunch of models and algorithms, but an universally acceptable solution approach covering the practicability is still lacking.

In this paper, we consider the evacuation problems with dynamic flows over time. The dynamic network problems with an objective of maximizing the flows at every point of time and minimizing the total evacuation time are studied. The classical models and analytical solution techniques are summarized and the recent results with extended solutions are also stated compactly. These results with illustrated examples give insights into the current issues of network flows over time and open new directions of further research in evacuation planning.

Acknowledgements The author acknowledges the partial support of DAAD under the partnership program between the departments of mathematics at Tribhuvan University, Nepal, Mindanao State University-IIT, Philippines and University of Kaiserslautern, Germany, entitled "Graph Theory and Optimization with Applications in Industry and Society (GraTho)". The author also thanks the support of Alexander von Humboldt Foundation under the AvH Research Group Linkage Progremme at TU Bergakedemie Freiberg (August 18-November 14, 2016). 


\section{References}

[1] Baumann N and Skutella M (2009), Earliest arrival flows with multiple sources. Mathematics of Operations Research 34:499-512.

[2] Burkard RE, Dlaska K and Klinz B (1993), The quickest flow problem. ZOR-Methods and Models of Operations Research 37:31-58.

[3] Dhamala TN (2015), A survey on models and algorithms for discrete evacuation planning network problems. Journal of Industrial and Management Optimization 11:265-289.

[4] Dhamala TN and Pyakurel U (2013), Earliest arrival contraflow problem on series-parallel graphs. International Journal of Operations Research, 10:1-13.

[5] Fleischer LK and Tardos E (1998), Efficient continuous-time dynamic network flow algorithms. Operations Research Letters 23:71-80.

[6] Ford LR and Fulkerson DR (1958), Constructing maximal dynamic flows from static flows. Operations Research 6:419-433.

[7] Ford LR and Fulkerson DR (1962), Flows in networks, Princeton University Press, Princeton, New Jersey.

[8] Gale D (1959). Transient flows in networks. Michigan Mathematical Journal 6:59-63.

[9] Hamacher HW and Tjandra SA (2002). Mathematical modeling of evacuation problems: A state of the art. In: M. Schreckenberger and S.D. Sharma (Eds.), Pedestrain and Evacuation Dynamics, Springer, Berlin, Heidelberg, 227-266.

[10] Hoppe B and Tardos E (2000). The quickest transshipment problem. Mathematics of Operations Research 25:36-62.

[11] Pyakurel U, Dhamala TN and Dempe S (2017), Efficient continuous contraflow algorithms for evacuation planning problems. Annals of Operations Research (ANOR), 254:335-364.

[12] Pyakurel U (2016), Evacuation planning problem with contraflow approach. PhD Thesis, IOST, Tribhuvan University, Nepal.

[13] Pyakurel U and Dhamala TN (2015). Models and algorithms on contraflow evacuation planning network problems. International Journal of Operations Research, 12:36-46.

[14] Pyakurel U and Dhamala TN (2017). Evacuation planning by earliest arrival contraflow. Journal of Industrial and Management Optimization 13:487-501.

[15] Pyakurel U and Dhamala TN (2016a), Continuous time dynamic contraflow models and algorithms. Advance in Operations Research; Article ID 368587, 1-7.

[16] Pyakurel U and Dhamala TN (2017), Continuous dynamic contraflow approach for evacuation planning. Annals of Operation Research (ANOR), 253: 573-598.

[17] Pyakurel U, Hamacher HW and Dhamala TN (2014), Generalized maximum dynamic contraflow on lossy network. International Journal of Operations Research Nepal 3: 27-44.

[18] Rebennack S, Arulselvan A, Elefteriadou L and Pardalos PM (2010), Complexity analysis for maximum flow problems with arc reversals. Journal of Combinatorial Optimization 19:200-216. 